\title{
Analyzing the Higher Order Structure of Proteins with Conformer- Selective Ultraviolet Photodissociation
}

\author{
Stephan Warnke ${ }^{1}$, Gert von Helden ${ }^{1}$, and Kevin Pagel $^{1,2}$ \\ ${ }^{1}$ Fritz Haber Institute of the Max Planck Society, Faradayweg 4-6, 14195 Berlin, Germany. \\ ${ }^{2}$ Freie Universität Berlin, Institute of Chemistry and Biochemistry, Takustraße 3, 14195 Berlin, Germany
}

\section{Correspondence}

Prof. Dr. Kevin Pagel, Freie Universität Berlin, Institute of Chemistry and Biochemistry - Organic Chemistry, Takustraße 3, 14195 Berlin, Germany. Email: kevin.pagel@fu-berlin.de

\footnotetext{
Abbreviations: Arrival time distribution (ATD); collision cross section (CCS); ion mobility spectrometry (IMS); ultraviolet photodissociation (UVPD)

Keywords: electrospray ionization mass spectrometry (ESI-MS); ion mobility spectrometry (IMS); peptide sequencing; topdown proteomics; ultraviolet photodissociation (UVPD)

Total number of words: 5137
} 
ABSTRACT: The top-down approach in protein sequencing requires simple methods in which the analyte can be readily dissociated at every position along the backbone. In this context, ultraviolet photodissociation (UVPD) recently emerged as a promising tool because, in contrast to slow heating techniques such as collision induced dissociation (CID), the absorption of UV light is followed by a rather statistically distributed cleavage of backbone bonds. As a result, nearly complete sequence coverage can be obtained. It is well-known, however, that gas-phase proteins can adopt a variety of different, sometimes coexisting conformations and the influence of this structural diversity on the UVPD fragmentation behavior is not clear.

Using ion mobility-UVPD-mass spectrometry we recently showed that UVPD is sensitive to the higher order structure of gasphase proteins. In particular, the cis/trans isomerization of certain proline peptide bonds was shown to significantly influence the UVPD fragmentation pattern of two extended conformers of $11^{+}$ubiquitin. Building on these results, we here provide conformer-selective UVPD data for $7^{+}$ubiquitin ions, which are known to be present in a much more diverse and wider ensemble of different structures, ranging from very compact to highly extended species. Our data show that certain conformers fall into groups with similar UVPD fragmentation pattern. Surprisingly, however, the conformers within each group can differ tremendously in their collision cross section. This indicates that the multiple coexisting conformations typically observed for $7^{+}$ ubiquitin are caused by a few, not easily inter-convertible, subpopulations. 


\section{Introduction}

The ability to dissociate peptide and protein ions in the gas phase of a mass spectrometer is essential for the vast majority of proteomics experiments performed today. For this purpose, simple workflows, which deliver a high content of sequence information are desirable. The workhorse technique in top-down protein sequencing is tandem-mass spectrometry (MS-MS) in combination with collision induced dissociation (CID).[1] However, peptide bond cleavages do not occur randomly along the sequence but rather at specific positions when the molecule is slowly heated during CID. As a result, often only little sequence coverage and incomplete information about the primary structure is obtained, which in turn can be problematic, especially for the characterization of unknown compounds.

Because of this limitation, over the past few years electron capture- and electron transfer dissociation (ECD/ETD) have received increased attention, since in those methods, cleavages occur less selective along the sequence and therefore greatly increased sequence information can be obtained.[2,3] Unlike in collisional heating methods, noncovalent interactions can be retained after dissociation of the backbone using ECD/ETD, which can facilitate the investigation of protein complexes and higher order protein structures.[3-5] However, the ECD/ETD process is most efficient for precursor ions with high charge density $[6,7]$, which, on the other hand, often leads to unfolding of the gas-phase protein. As a consequence, compact, more native-like gas-phase ions are usually difficult to investigate by ECD/ETD. A dissociation method, which recently emerged as a promising alternative is based on the absorption of UV photons by the amide backbone and subsequent dissociation [8]. During this ultraviolet photodissociation (UVPD), peptide bond cleavages occur rather statistically over the entire amino acid sequence, which can yield nearly complete sequence coverage for intact proteins [9-12] and even noncovalent protein assemblies.[13]

Depending on solvent conditions and the amount of charged sites, proteins can adopt a multitude of different conformations in the gas phase. Low charge states typically adopt compact, native-like structures governed by non-covalent, intramolecular interactions, whereas the protein tends to unfold to elongated structures, dominated by charge repulsion when the charge state of the molecule is increased [14]. Such structural differences can, for example, be analyzed by ion mobility spectrometry (IMS). This technique makes use of the different drift velocities that protein conformers exhibit when they drift through an inert buffer gas under the influence of a weak electric field [15-18]. The measured drift time can be converted into an angular averaged collision cross section (CCS), which is a measure for the size and shape of the ion [19]. Moreover, the method can be easily combined with MS (IM-MS), which allows the simultaneous analysis of $\mathrm{m} / \mathrm{z}$ and CCS.

Using IM-MS in conjunction with UVPD, we recently demonstrated that higher order structure of a protein can drastically influence its photofragmentation behavior at $193 \mathrm{~nm}$ [20]. The UVPD spectra of two different elongated structures of the 8.5 $\mathrm{kDa}$ protein ubiquitin in charge state $11^{+}$showed a significant variation in the fragment pattern, which was attributed to a difference in the intramolecular hydrogen-bond pattern as a consequence of cis/trans isomerization of a single peptidyl-prolyl bond. Here, we build on these results and present conformer selective UVPD data for ubiquitin $7^{+}$ions, which are known to adopt a large variety of compact and extended conformations in the gas phase. Our data indicate that UVPD is also structure sensitive for this conformationally much more diverse charge state. Additionally, we find evidence for a connection between 
certain conformers, which show similar UVPD dissociation patterns even if they exhibit vastly different CCSs. From that we conclude that groups of structures with considerably different CCSs but similar structural features within one group exist.

\section{Materials and Methods}

\subsection{Samples}

Human ubiquitin ( 8.5 kDa) was purchased from R\&D Systems (Wiesbaden, Germany) and used without further purification. Solvents were purchased from Sigma-Aldrich (Taufkirchen, Germany). For electrospray ionization, an aqueous $1 \mathrm{mM}$ ubiquitin stock solution was diluted with water/methanol v/v 50/50 to yield a concentration of $10 \mu \mathrm{M}$. For (nano) electrospray ionization $\sim 5 \mu 1$ of sample were loaded into a $\mathrm{Pd} / \mathrm{Pt}$ coated borosilicate capillary prepared in-house and a voltage of $0.8-1.0 \mathrm{kV}$ was applied [21].

\subsection{Experimental setup for conformer-resolved ultraviolet photodissociation experiments}

The ion mobility method has been described in detail previously [15-18]. To preselect gas-phase protein conformers prior to UV photodissociation (UVPD), an in-house built drift tube (DT) ion mobility-mass spectrometer similar to one described previously was used (Figure 1 B) [20, 22]. Ions are generated in a nano-electrospray ionization source (nESI) [23] and transferred into the vacuum. An electrodynamic ion funnel collects ions and releases ion pulses into the drift region where they drift through helium buffer gas ( $\sim 5$ mbar) under the influence of a weak electric field $(\sim 10 \mathrm{~V} / \mathrm{cm})$. A second ion funnel guides the now conformer separated but diffusionally spread ion clouds through a conductance limit into a differentially pumped region where ion guides transport them into ultra high vacuum. Here, a quadrupole mass filter selects ions of a specific $\mathrm{m} / \mathrm{z}$ value. Arrival time distributions (ATDs) can then be recorded by measuring the time dependent ion current after release of the ion trap.

For the acquisition of conformer-selective UVPD spectra, ions of a narrow drift time window (100 $\mu$ s, corresponding to a narrow collision cross section distribution) can be selected by electrostatic deflection prior mass selection. While traversing a second quadrupole guide, the now conformer and $\mathrm{m} / \mathrm{z}$-selected ions are irradiated by a single unfocussed excimer laser pulse $(193 \mathrm{~nm}, \sim 1 \mathrm{~mJ})$ and the resulting fragments are detected by means of time-of-flight (ToF) MS.

In Figure $1 \mathrm{~A}$, a typical trigger pulse sequence for the conformer selective UVPD experiment is depicted. It is initiated by a 150 $\mu$ s ion gate pulse, followed by a $100 \mu$ s admittance window of the electrostatic deflection lens a few milliseconds later. By varying the delay $\Delta t_{l}$, ions of different drift times can be selected. By the time ions traverse the second quadrupole guide, the laser pulse is triggered which induces fragmentation over a wide $m / z$ range. An inevitable kinetic energy spread of the fragment ion cloud, accompanied by slightly differing flight times towards the ToF mass analyzer has to be accommodated by varying the delay $\Delta t_{3}(90-130 \mu \mathrm{s})$, which determines the moment of the ToF pulse. Subsequently, fragment spectra of different $\Delta t_{3}$ values are averaged to yield mass spectra of the full fragment range. 


\subsection{Fragment assignment}

The mass accuracy and resolving power $(\Delta m / m \sim 500)$ of the in-house built linear ToF mass analyzer attached to the drift-tube instrument does not allow for a straightforward UVPD fragment assignment. Instead, comparison of a list of possible fragments with high-resolution UVPD fragment spectra from earlier work $[12,20]$ (and unpublished data), as well as the observed CID fragmentation pattern from a high-resolution Waters Synapt G2-S instrument (see SI), helped to assign and understand the rather complex fragment spectra at least in part.

\subsection{Collision cross section determination}

For better comparison, the time axis of the arrival time distributions (ATDs) was converted to collision cross sections $\Omega$. For conversion of ATDs from DT IMS instruments, which utilizes a uniform electric field in the drift region, this can be achieved using the following equation [19]:

$\Omega=\frac{3 z e}{16 N} \sqrt{\frac{2 \pi}{\mu k_{B} T}} \frac{t_{D} E}{L} \frac{1013 T}{273.16 P}$

where $z$ and $e$ correspond to the charge state of the investigated ions and the elementary charge, $\mu$ is the reduced mass of the ion-buffer gas molecule system, $k_{B}$ and $T$ are the Boltzmann constant and the temperature, $t_{D}$ is the drift time, $E$ and $L$ are the electric field and the length of the drift cell, $N$ is the number density of the buffer gas molecules and $P$ is the pressure (in mbar). CCS values reported here are in good agreement with previously published values for ubiquitin [24, 25].

\section{Results and Discussion}

\subsection{Ion mobility-mass spectrometry of ubiquitin $7^{+}$}

Ubiquitin has been investigated extensively in solution and in the gas phase using a variety of techniques. In both environments, various distinct conformations, ranging from compact, globular to unfolded, extended structures have been observed [26-30]. As for most gas-phase proteins, the charge state of the ions was found to be most influential on the structure. Compact conformations require low charge states whereas more elongated structures are dominantly found for higher charge states. Moreover, recent studies have shown that the gas-phase conformation of ubiquitin is also closely linked to the solution conditions the ions originate from $[25,29,31,32]$. When electrosprayed from aqueous or more denaturing solvents, ubiquitin was shown to adopt compact, native-like conformations, as well as more elongated $\alpha$-helix rich structure (the so-called A-state) $[25,31]$, which are similar to those existing in the condensed phase.[26-28] Using IMS-based techniques, it was furthermore shown that there is a certain connection between distinct folded and unfolded structures of gas-phase ubiquitin, which was attributed to structural features that are preserved during the unfolding process [33].

In this study, we focus on ubiquitin ions of the intermediate charge state $7^{+}$in which attractive and repulsive intramolecular interactions are balanced and multiple different structures are observed simultaneously using IM-MS. For these ions, the distribution of gas-phase conformers strongly depends on instrument parameters such as source conditions and injection 
energy. At gentle conditions, a compact species with a CCS of $\sim 1000 \AA^{2}$ can be observed, which unfolds to intermediate and extended structures with CCSs ranging from $1200 \AA^{2}$ to $1600 \AA^{2}$ when the molecules are energized.

In Figure 2 typical arrival time distributions (ATDs) of ubiquitin $7^{+}$measured at two different trap conditions are shown with drift times converted to CCSs. At intermediate conditions, the ATD exhibits a variety of partially folded $\left(1200 \AA^{2}-1450 \AA^{2}\right)$ and extended structures $\left(>1450 \AA^{2}\right)$ all of which having been observed previously [31, 34]. A more compact conformer around $1000 \AA^{2}$ was also observed but not studied in detail here due to very low ion intensities. At harsher trap conditions at least two more extended structures were observed with CCSs of $\sim 1500 \AA^{2}$ and $\sim 1600 \AA^{2}$, respectively. The narrow peaks consecutively labeled from 1 to 9 are the slices of the ATDs that have been selected at the ion ejection lens and were transmitted for UV photodissociation.

\subsection{UV photodissociation of selected ubiquitin $7^{+}$conformers}

After drift time and $\mathrm{m} / \mathrm{z}$-selection, the isolated ions are subjected to a single unfocused $193 \mathrm{~nm}$ ArF excimer laser pulse $(\sim 1 \mathrm{~mJ})$ and fragment spectra are recorded for each selected conformer individually by averaging over 500 ToF pulses. The so obtained UV photofragment spectra of the nine different ubiquitin $7^{+}$structures from the ATD in Figure 2 are shown in Figure 3. The spectra are normalized to the intensity of the precursor ion signal and then equally scaled to the level of the fragment intensities. The roman numerals I to VIII denote the strongest ion signals. As expected from previous UVPD experiments [12, 20], a vast variety of different fragments is formed, which in turn results in rich fragment spectra with many, often only partially resolved signals. All ion signals, however, can be clearly distinguished from noise level, as can be seen exemplarily in Figure S1 of the supporting information (SI) where mass spectra of UV-irradiated and non-irradiated ions are compared. Many of the fragment signals in Figure 3 can be found in all nine spectra; others, however, are only present in some. Among the most apparent differences are the fragments labeled I, II and VIII, which are of higher intensities in the fragment spectra of the ATD slices labeled 5, 6, 7, and 9 than in the spectra labeled 1, 2, 3, 4, and 8. In addition the fragment $\mathbf{V}$ is of considerably higher intensity in the latter set of spectra.

Even without further assignment of the peaks, two aspects can already be concluded from the spectra in Figure 3 . First, ubiquitin $7^{+}$ions show a different UVPD fragmentation behavior depending on their conformation. This is interesting by itself and indicates that UVPD is structure-sensitive to a certain extend. In contrast, CID experiments on the same conformers showed no dependence on the structure and yielded almost identical fragment spectra, which is in good agreement with previous observations (for details see Figures S2/S3).[20, 35] Second, some of the fragmentation spectra along the conformer distribution are rather similar, which points to a link between certain conformers.

\subsection{Fragment assignment}

A deeper understanding of the observed dependence on conformation requires an unambiguous assignment of the obtained fragments. Due to the limited resolution of the mass spectrometer used here, however, such an assignment cannot be made purely on basis of the presented UVPD fragment spectra. Given that UVPD yields a vast variety of different fragments and considering the mass accuracy $(\mathrm{m} / \mathrm{z} \pm 1.5)$ and resolution $(\Delta \mathrm{m} / \mathrm{m} \sim 500)$ of the instrument, up to five distinct photofragments can 
be hypothetically assigned to the signals labeled I to VIII (Table 1). To narrow down the list of possible candidates, unambiguously identified UVPD fragments of non-conformer selected ubiquitin $10^{+}$and $11^{+}$ions from high-resolution experiments [12] (unpublished data) were compared with the fragments in Table 1. For each signal I to VIII, at least two fragment candidates have been previously observed in UVPD of $10^{+}$and $11^{+}$ubiquitin. These fragments are marked with an $\mathbf{x}$ or, if the fragments are particularly prominent in the reference spectra, with $\mathbf{x x}$ in Table 1. It is interesting to note that for each of the features I to VIII at least one fragment is observed that stems from cleavage of a peptide bond involving one of the three proline residues that are present at positions 19,37 , and 38 in the sequence of ubiquitin. In addition, the fragments involving proline residues are generally among the most prominent signals in the UVPD spectra of the higher charged species (unpublished data).[12]

Comparison with other dissociation methods can yield further information about the identity of the observed UVPD fragments. Fragment spectra obtained through dissociation techniques involving slow heating (such as CID), are largely dominated by $b$ and $y$ type fragments stemming from cleavages $\mathrm{N}$-terminal to proline or $\mathrm{C}$-terminal to acidic residues.[12, 13, 36-38] This trend is also observed in the CID spectra of ubiquitin $7^{+}$and leads to prominent series of C-terminal $y$-type fragments, stemming from cleavages of the peptide bonds between $\mathrm{Glu}^{18}-\mathrm{Pro}^{19}$ and preceding residues (for details see SI). Intriguingly, five Cterminal $y$-type CID fragments are also among the previously observed UV photofragments in Table 1. Most of these fragments, namely $y_{58}\left(5^{+}, 6^{+}\right), y_{40}\left(4^{+}\right)$, and $y_{59}\left(5^{+}\right)$, stem from cleavage of peptide bonds preceding proline residues. Unlike in the UVPD experiments, however, no significant differences between the CID fragment spectra of different conformers are observed. A dissociation method that was previously shown to depend on the higher order structure of ubiquitin is ECD.[3, 39, 40] Using this dissociation technique for ubiquitin $7^{+}$, however, mainly leads to $c$ - and $z$-type ions [39], none of which having been observed in previous UVPD experiments of ubiquitin $10^{+}$and $11^{+}$(for details see SI).

Taken together, there is still a certain ambiguity in the identification of possible fragments for the eight most prominent signals in Figure 3. However, there is clear evidence for a prevalence of fragments stemming from cleavages of peptide bonds involving proline residues.[20] Such a proline-effect is well known for slow heating techniques such as CID [36, 38], but was previously shown to be not significant in UVPD.[10] In addition, the tentative assignment made here only involves the eight most prominent of the hundreds of different fragments observed in the experiment and does, thus, not contradict earlier studies where rather non-specific backbone cleavage is found upon photodissociation at $193 \mathrm{~nm}$.

\subsection{Connection between conformers}

To gain more insight into the apparent link between certain structures, the intensities of the fragments that exhibit noticeable intensity variations along the conformer distribution are plotted in Figure 4. For clarity, open and solid symbols are used, indicating two different types of curve progressions. The fragments labeled I, II and VIII in Figure 3, each increase in intensities for conformers 5, 6, 7, and 9, whereas the opposite is the case for the fragments labeled V, VI, and VII, which are of higher intensities in the fragment spectra of the conformers $1,2,3,4$, and 8 . This observation supports the hypothesis that certain structures are connected. Accordingly, the nine different structures of ubiquitin $7^{+}$can be classified into two groups of conformers with similar photofragmentation patterns, namely structures 1-2-3-4-8 (group A) and structures 5-6-7-9 (group B). 
The fact that the photofragmentation patterns of two distinct conformers are different must be a result of a difference in structure. One premise for the coexistence of multiple structures at a temperature of $300 \mathrm{~K}$ is the existence of sufficiently high energy barriers that separates their corresponding conformational space and prevents group A and group B structures from interconverting on the timescale of the experiment. In solution studies on ubiquitin, cis/trans isomerization of peptide bonds involving loop and turn-inducing proline residues has been identified to form such a barrier, which is typically around $50-80$ $\mathrm{kJ} / \mathrm{mol}$ [41]. Especially the residue Pro $^{19}$ was found to be of particular importance for the protein structure [42], and we showed that cis/trans isomerization of the peptide bond preceding the residue Pro ${ }^{19}$ is likely the reason for coexisting gas-phase structures of $11^{+}$ubiquitin [20]. Similar conclusions have been drawn for smaller proline containing model peptides where multiple coexisting gas-phase conformations were attributed to isomerization of peptidyl-prolyl bonds [43, 44].

Our tentative fragment assignment suggests that for ubiquitin $7^{+}$, like in the $11^{+}$case, many differences in fragment identities and relative abundances arise from cleavage around $\mathrm{Xxx}$-Pro peptide bonds, which leads to the assumption that isomerization of these peptide bonds plays an important role for the $7^{+}$ions as well. The two different structural groups A and B are, therefore, likely to differ in the isomerization state of peptidyl-prolyl bonds. Such a subtle difference in local structure, on the other hand, can severely influence the entire intramolecular hydrogen bonding network of the protein.[20] In ECD and ETD experiments [4, 40,45], as well as vacuum UVPD on protein-ligand complexes [46] it was found that the retention of noncovalent interactions after cleavage of the backbone can lead to distinct fragmentation patterns. It is not unlikely, that similar effects are responsible for the differences in the UVPD spectra observed here.

Fragment spectra from low energy CID experiments do not contain information about differences in higher order structure.[35] This is not surprising because the internal energy of the molecule is increased gradually with each collision until it reaches the dissociation threshold, which typically exceeds the barrier for cis/trans isomerization of Xxx-Pro peptide bonds [47]. The situation is different, however, when the unfolding rather than the dissociation of the molecule is monitored after subjection to collisions with inert gas molecules. In a recent series of studies on collision induced unfolding (CIU) of different gas-phase conformations of ubiquitin $7^{+}$, it was concluded that partially folded and unfolded structures can retain certain structural aspects of the compact species from which they originated [31,33]. In other words, certain structures are linked over an unfolding path, with shared structural motifs. Our results support this hypothesis and indicate that the similarities in the photofragmentation pattern within one group arise from shared structural features, which are maintained over the group's entire conformational space (i.e. 1-2-3-4-8 for group A and 5-6-7-9 for group B).

\section{Concluding Remarks}

UVPD recently emerged as a promising technique for peptide and protein sequencing because nearly complete sequence coverage can be achieved within a relatively simple experiment. Using a combined IM-MS-UVPD-ToF-MS approach where ions can be size and $\mathrm{m} / \mathrm{z}$ selected before photodissociation $(193 \mathrm{~nm})$, we show here, that UVPD is sensitive to the underlying higher order structure of conformationally highly diverse $7^{+}$ubiquitin ions. Considerable differences in fragment identities and abundances were observed in the photofragment spectra of different pre-selected conformations. These results are in good 
agreement with previously reported data on two extended conformers of $11^{+}$ubiquitin, which showed a significantly different UVPD fragmentation behavior.

At the current state, however, our data also imply that specific conformers not necessarily yield unique UVPD fragment spectra. Instead, we find repeating fragment patterns, which indicate that certain structures within the ensemble are closely connected. Based on these results, the conformers of $7^{+}$ubiquitin can be categorized into two groups, A and B, each of which exhibiting various structures with different CCSs but a similar UVPD fragmentation behavior. This leads us to the assumption that, despite their difference in CCS, the conformers within one group share a common structural feature, which is retained over the entire gas-phase unfolding pathway, while a relatively large energy barrier prevents interconversion between both groups on the timescale of the experiment. In our previous study on $11^{+}$ubiquitin, cis/trans isomerization of Xxx-Pro peptide bonds was identified as a possible cause for the varying photodissociation pattern. Even though the limited mass resolution in the photofragment spectra reported here does not allow us to unambiguously assign all fragments, it is likely that the hereobserved differences in the UVPD spectra are also originating from cis/trans isomerization of peptidyl-prolyl bonds.

Seen from a broader perspective, our data imply that the conformational families often observed for gas-phase proteins, are in fact groups of dynamically interconverting structures that are separated by a relatively large energy barrier. While UVPD is not sensitive to the conformational aspects within one group, it can well be used to distinguish between different groups - for example to identify proline cis/trans isomers, which are known to have a significant impact on the folding of proteins in both solution and gas phase. 


\section{ACKNOWLEDGMENT}

The authors thank Michael T. Bowers for continuing support and helpful discussions. Jennifer S. Brodbelt is gratefully acknowledged for making available unpublished high-resolution UVPD data.

The authors have declared no conflict of interest 


\section{References}

[1] Papayannopoulos, I. A., The interpretation of collision-induced dissociation tandem mass spectra of peptides. Mass Spectrom. Rev. 1995, 14, 49-73.

[2] Syka, J. E., Coon, J. J., Schroeder, M. J., Shabanowitz, J., Hunt, D. F., Peptide and protein sequence analysis by electron transfer dissociation mass spectrometry. Proc. Natl. Acad. Sci. U. S. A. 2004, 101, 9528-9533.

[3] Zubarev, R. A., Kelleher, N. L., McLafferty, F. W., Electron Capture Dissociation of Multiply Charged Protein Cations. A Nonergodic Process. J. Am. Chem. Soc. 1998, 120, 3265-3266.

[4] Lermyte, F., et al., ETD Allows for Native Surface Mapping of a $150 \mathrm{kDa}$ Noncovalent Complex on a Commercial QTWIMS-TOF Instrument. J. Am. Soc. Mass Spectrom. 2014, 25, 343-350.

[5] Zhang, H., Cui, W., Gross, M. L., Blankenship, R. E., Native mass spectrometry of photosynthetic pigment-protein complexes. FEBS Lett. 2013, 587, 1012-1020.

[6] Ledvina, A. R., et al., Infrared photoactivation reduces peptide folding and hydrogen-atom migration following ETD tandem mass spectrometry. Angew. Chem. Int. Ed. Engl. 2009, 48, 8526-8528.

[7] Horn, D. M., Breuker, K., Frank, A. J., McLafferty, F. W., Kinetic Intermediates in the Folding of Gaseous Protein Ions Characterized by Electron Capture Dissociation Mass Spectrometry. J. Am. Chem. Soc. 2001, 123, 9792-9799.

[8] Ly, T., Julian, R. R., Ultraviolet photodissociation: Developments towards applications for mass-spectrometry-based proteomics. Angew. Chem. Int. Ed. 2009, 48, 7130-7137.

[9] Guan, Z., et al., $193 \mathrm{~nm}$ photodissociation of larger multiply-charged biomolecules. Int. J. Mass Spectrom. Ion Processes $1996,157-158,357-364$.

[10] Choi, K. M., et al., Characteristics of photodissociation at $193 \mathrm{~nm}$ of singly protonated peptides generated by matrixassisted laser desorption ionization (MALDI). J. Am. Soc. Mass Spectrom. 2006, 17, 1643-1653.

[11] Reilly, J. P., Ultraviolet photofragmentation of biomolecular ions. Mass Spectrom. Rev. 2009, 28, 425-447.

[12] Shaw, J. B., et al., Complete protein characterization using top-down mass spectrometry and ultraviolet photodissociation. $J$. Am. Chem. Soc. 2013, 135, 12646-12651.

[13] O'Brien, J. P., Li, W., Zhang, Y., Brodbelt, J. S., Characterization of native protein complexes using ultraviolet photodissociation mass spectrometry. J. Am. Chem. Soc. 2014, 136, 12920-12928.

[14] Hall, Z., Robinson, C. V., Do charge state signatures guarantee protein conformations? J Am Soc Mass Spectrom 2012, 23, 1161-1168.

[15] Wyttenbach, T., Bowers, M., in: Schalley, C. A. (Ed.), Modern Mass Spectrometry, Springer Berlin Heidelberg 2003, pp. 207-232.

[16] Bohrer, B. C., Merenbloom, S. I., Koeniger, S. L., Hilderbrand, A. E., Clemmer, D. E., Biomolecule Analysis by Ion Mobility Spectrometry. Annual Review of Analytical Chemistry 2008, 1, 293-327.

[17] Uetrecht, C., Rose, R. J., van Duijn, E., Lorenzen, K., Heck, A. J. R., Ion mobility mass spectrometry of proteins and protein assemblies. Chemical Society Reviews 2010, 39, 1633-1655.

[18] Lanucara, F., Holman, S. W., Gray, C. J., Eyers, C. E., The power of ion mobility-mass spectrometry for structural characterization and the study of conformational dynamics. Nat. Chem. 2014, 6, 281-294. 
[20] Warnke, S., Baldauf, C., Bowers, M. T., Pagel, K., von Helden, G., Photodissociation of conformer-selected ubiquitin ions reveals site-specific cis/trans isomerization of proline peptide bonds. J. Am. Chem. Soc. 2014, 136, 10308-10314.

[21] Hernandez, H., Robinson, C. V., Determining the stoichiometry and interactions of macromolecular assemblies from mass spectrometry. Nat Protoc 2007, 2, 715-726.

[22] Kemper, P. R., Dupuis, N. F., Bowers, M. T., A new, higher resolution, ion mobility mass spectrometer. Int. J. Mass Spectrom. 2009, 287, 46-57.

[23] Wilm, M., Mann, M., Analytical properties of the nanoelectrospray ion source. Anal. Chem. 1996, 68, 1-8.

[24] Myung, S., Badman, E. R., Lee, Y. J., Clemmer, D. E., Structural transitions of electrosprayed ubiquitin ions stored in an ion trap over $10 \mathrm{~ms}$ to 30 s. J. Phys. Chem. A 2002, 106, 9976-9982.

[25] Segev, E., Wyttenbach, T., Bowers, M. T., Gerber, R. B., Conformational evolution of ubiquitin ions in electrospray mass spectrometry: molecular dynamics simulations at gradually increasing temperatures. Phys. Chem. Chem. Phys. 2008, 10, $3077-3082$

[26] Wilkinson, K. D., Mayer, A. N., Alcohol-induced conformational changes of ubiquitin. Arch. Biochem. Biophys. 1986, 250, 390-399.

[27] Vijay-Kumar, S., Bugg, C. E., Cook, W. J., Structure of ubiquitin refined at 1.8§̊ resolution. J. Mol. Biol. 1987, 194, 531544.

[28] Brutscher, B., Bruschweiler, R., Ernst, R. R., Backbone dynamics and structural characterization of the partially folded A state of ubiquitin by 1H, 13C, and 15N nuclear magnetic resonance spectroscopy. Biochemistry 1997, 36, 13043-13053.

[29] Li, J., Taraszka, J. A., Counterman, A. E., Clemmer, D. E., Influence of solvent composition and capillary temperature on the conformations of electrosprayed ions: unfolding of compact ubiquitin conformers from pseudonative and denatured solutions. Int. J. Mass Spectrom. 1999, 185-187, 37-47.

[30] Wyttenbach, T., Pierson, N. A., Clemmer, D. E., Bowers, M. T., Ion mobility analysis of molecular dynamics. Annu. Rev. Phys. Chem. 2014, 65, 175-196.

[31] Shi, H., Atlasevich, N., Merenbloom, S. I., Clemmer, D. E., Solution dependence of the collisional activation of ubiquitin $[\mathrm{M}+7 \mathrm{H}]$ ions. J Am Soc Mass Spectrom 2014.

[32] Shi, H., Clemmer, D. E., Evidence for two new solution states of ubiquitin by IMS-MS analysis. J. Phys. Chem. B 2014, $118,3498-3506$

[33] Koeniger, S. L., Merenbloom, S. I., Sevugarajan, S., Clemmer, D. E., Transfer of structural elements from compact to extended states in unsolvated ubiquitin. J. Am. Chem. Soc. 2006, 128, 11713-11719.

[34] Wyttenbach, T., Bowers, M. T., Structural stability from solution to the gas phase: native solution structure of ubiquitin survives analysis in a solvent-free ion mobility-mass spectrometry environment. J. Phys. Chem. B 2011, 115, 12266-12275.

[35] Badman, E. R., Hoaglund-Hyzer, C. S., Clemmer, D. E., Dissociation of different conformations of ubiquitin ions. J. Am. Soc. Mass Spectrom. 2002, 13, 719-723.

[36] Vaisar, T., Urban, J., Probing Proline Effect in CID of Protonated Peptides. J. Mass Spectrom. 1996, 31, 1185-1187.

[37] Reid, G. E., Wu, J., Chrisman, P. A., Wells, J. M., McLuckey, S. A., Charge-state-dependent sequence analysis of protonated ubiquitin ions via ion trap tandem mass spectrometry. Anal. Chem. 2001, 73, 3274-3281. 
[38] Breci, L. A., Tabb, D. L., Yates, J. R., 3rd, Wysocki, V. H., Cleavage N-terminal to proline: analysis of a database of peptide tandem mass spectra. Anal. Chem. 2003, 75, 1963-1971.

[39] Breuker, K., Oh, H., Horn, D. M., Cerda, B. A., McLafferty, F. W., Detailed Unfolding and Folding of Gaseous Ubiquitin Ions Characterized by Electron Capture Dissociation. J. Am. Chem. Soc. 2002, 124, 6407-6420.

[40] Skinner, O. S., McLafferty, F. W., Breuker, K., How ubiquitin unfolds after transfer into the gas phase. J. Am. Soc. Mass Spectrom. 2012, 23, 1011-1014.

[41] Pal, D., Chakrabarti, P., Cis peptide bonds in proteins: residues involved, their conformations, interactions and locations. $J$. Mol. Biol. 1999, 294, 271-288.

[42] Briggs, M. S., Roder, H., Early hydrogen-bonding events in the folding reactions of ubiquitin. Proc. Natl. Acad. Sci. U. S. A. $1992,89,2027-2021$.

[43] Counterman, A. E., Clemmer, D. E., Cis-trans signatures of proline-containing tryptic peptides in the gas phase. Anal. Chem. 2002, 74, 1946-1951.

[44] Pierson, N. A., Chen, L., Russell, D. H., Clemmer, D. E., Cis-trans isomerizations of proline residues are key to bradykinin conformations. J. Am. Chem. Soc. 2013, 135, 3186-3192

[45] Zhang, H., Cui, W., Wen, J., Blankenship, R. E., Gross, M. L., Native electrospray and electron-capture dissociation FTICR mass spectrometry for top-down studies of protein assemblies. Anal. Chem. 2011, 83, 5598-5606.

[46] Canon, F., et al., Photodissociation and dissociative photoionization mass spectrometry of proteins and noncovalent proteinligand complexes. Angew. Chem. Int. Ed. Engl. 2013, 52, 8377-8381.

[47] Pierson, N. A., Clemmer, D. E., An IMS-IMS threshold method for semi-quantitative determination of activation barriers: Interconversion of proline cis $\leftrightarrow$ trans forms in triply protonated bradykinin. Int. J. Mass Spectrom. 2014. 


\section{Figures}

A
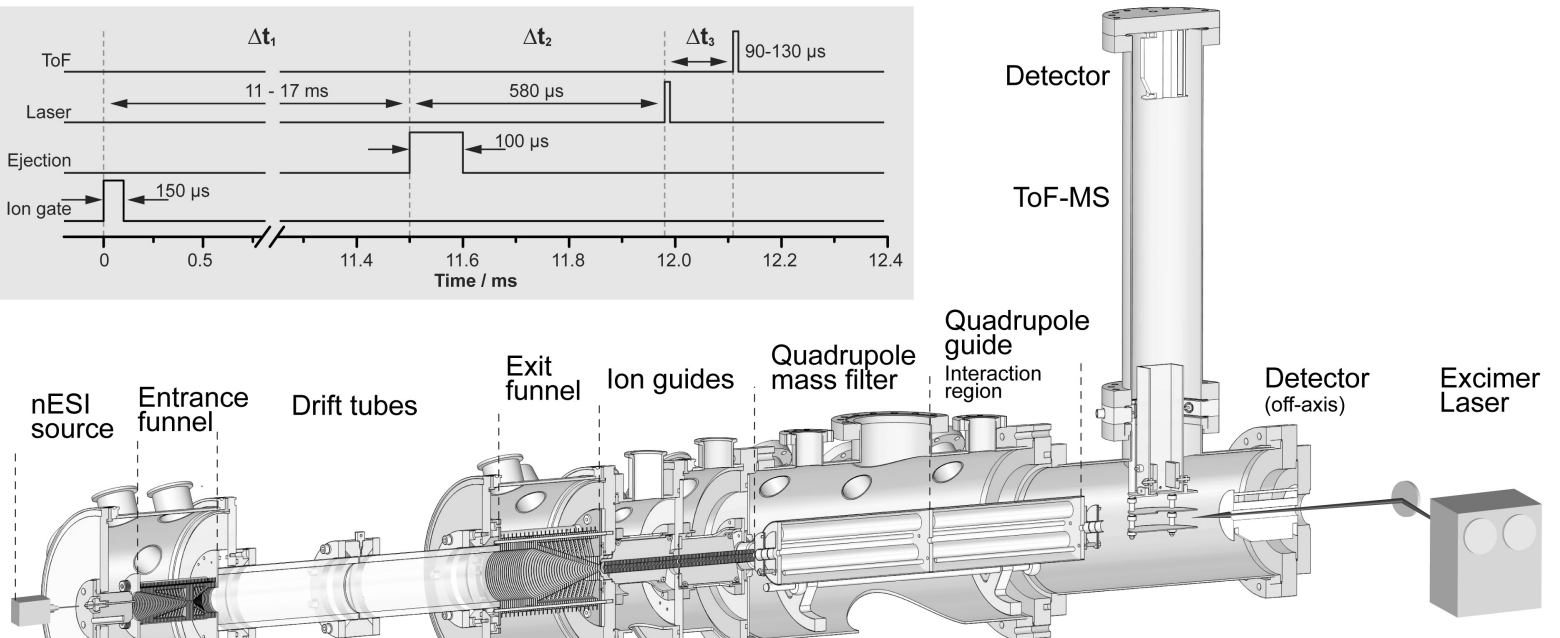

B

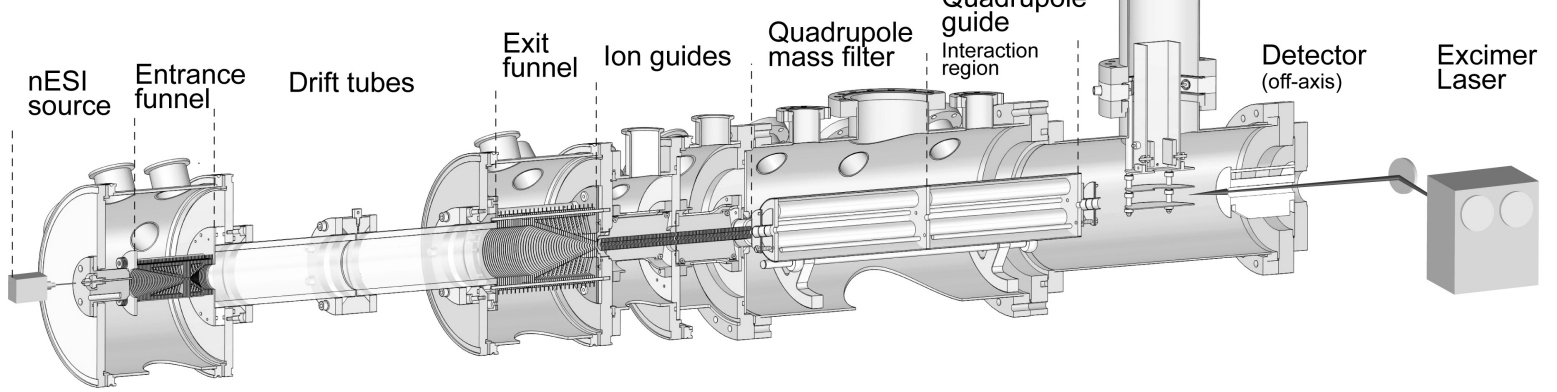

Figure 1: (A) Typical pulse sequence as applied for conformer-selective UVPD measurements at the drift tube IM-MS setup.

(B) Schematic diagram of the utilized instrument. After size and mass selection, ions are irradiated with a single excimer laser pulse and fragments are detected in the time-of-flight mass spectrometer.



Figure 2: Arrival time distributions (ATDs) of ubiquitin $7^{+}$at two different trap settings. The time axis was converted to collision cross section (CCS). The narrow peaks labeled consecutively from 1 to 9 are the selected portions of the ATDs transmitted for UV photodissociation. A previously reported more compact conformation $\left(\sim 1000 \AA^{2}\right)$ was not studied within this work. 


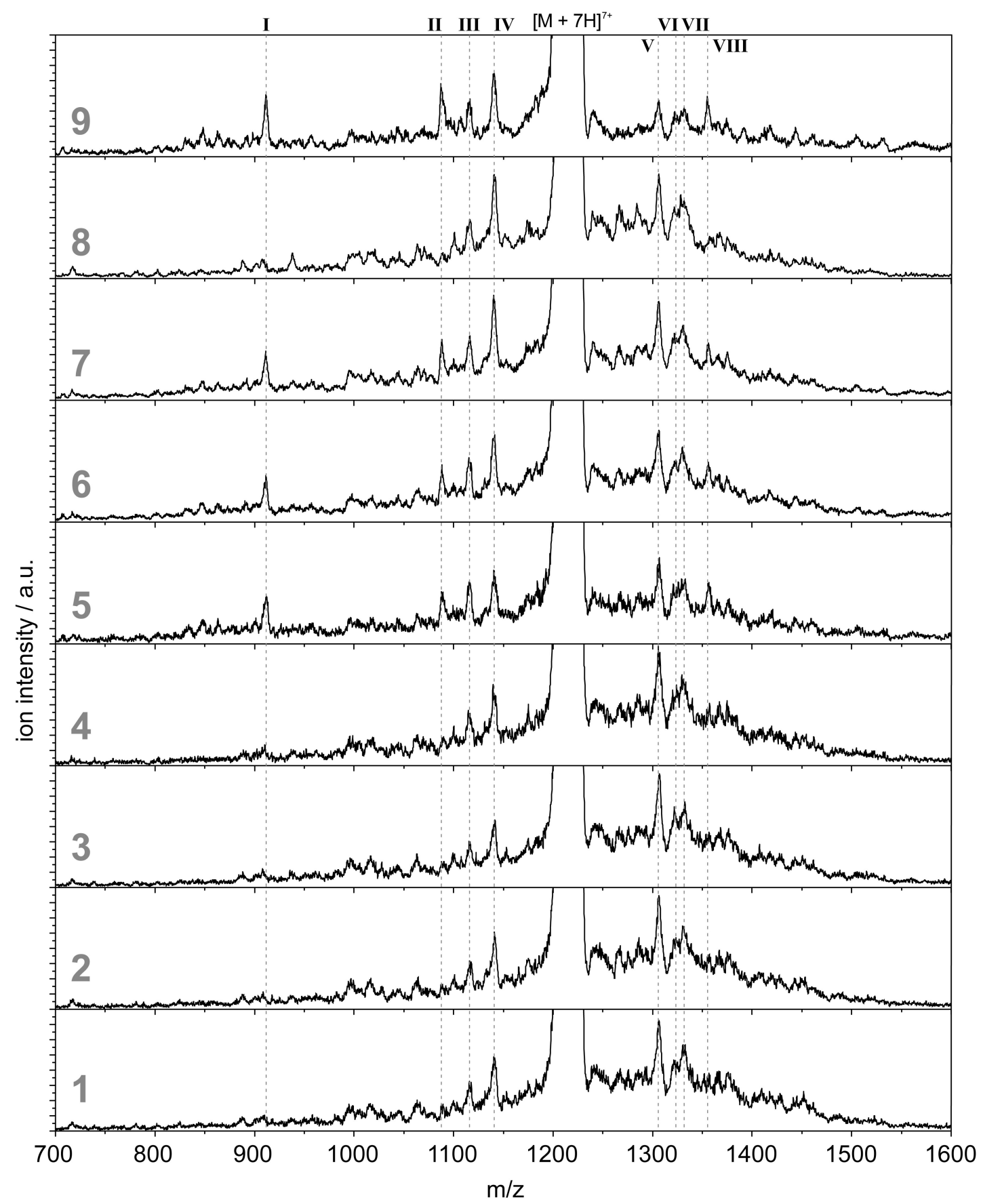

Figure 3: UVPD fragment spectra of the selected portions of the ATD labeled 1 to 9 in Figure 2. The spectra are normalized to the intensity of the precursor ion signal and then equally scaled to the level of the fragments. The dashed lines highlight the most prominent signals I - VIII. Conformers that occur at both trap settings (Figure 2) yield identical UVPD spectra. 


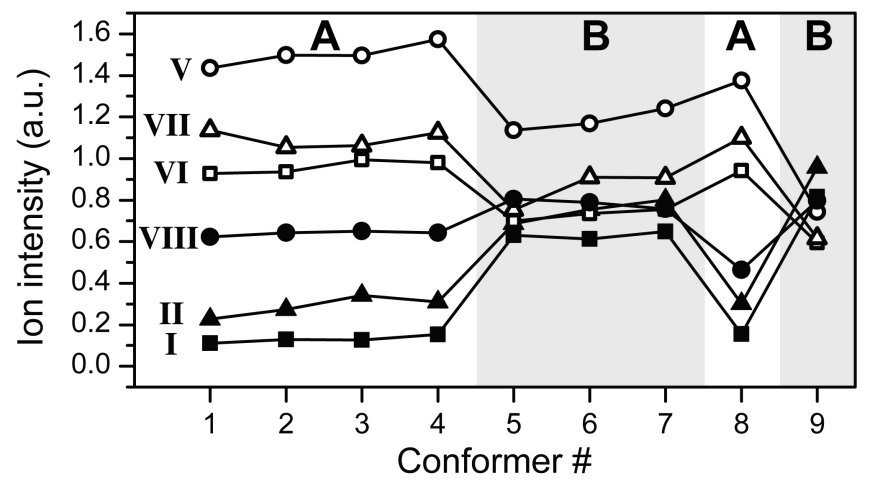

Figure 4: Dependence on conformation of the most abundant UVPD fragments. The signals labeled III and IV in Figure 3 show only little variation and are not included here. 
Table 1

\begin{tabular}{|c|c|c|c|c|c|}
\hline Label & $\begin{array}{l}m / z \\
\text { (exp.) }\end{array}$ & $\begin{array}{l}m / z \\
\text { (th.) }\end{array}$ & Type & $\begin{array}{l}\text { Sequence } \\
\text { position }\end{array}$ & $\begin{array}{l}\text { prev. } \\
\text { observed }^{\text {a }}\end{array}$ \\
\hline \multirow{5}{*}{ I } & \multirow{5}{*}{912.8} & 912.1 & $c_{16}$ & 16 & - \\
\hline & & 913.7 & $y_{48}$ & 29 & $\mathbf{x}$ \\
\hline & & 913.8 & $y_{40}$ & 37 & $\mathbf{x x}$ \\
\hline & & 914.3 & $b_{41}$ & 41 & - \\
\hline & & 914.4 & $b_{49}$ & 49 & - \\
\hline \multirow{2}{*}{ II } & \multirow{2}{*}{1089.0} & 1089.2 & $z_{38}$ & 38 & $\mathbf{x x}$ \\
\hline & & 1089.6 & $y_{58}$ & 19 & $\mathbf{x x}$ \\
\hline \multirow{4}{*}{ III } & \multirow{4}{*}{1118.1} & 1116.7 & $c_{30}$ & 47 & - \\
\hline & & 1117.4 & $z_{10}$ & 67 & $\mathbf{x}$ \\
\hline & & 1117.8 & $y_{39}$ & 38 & $\mathbf{x}$ \\
\hline & & 1118.4 & $c_{20}$ & 20 & - \\
\hline \multirow{5}{*}{ IV } & \multirow{5}{*}{1141.4} & 1141.3 & $x_{50}$ & 27 & - \\
\hline & & 1142.1 & $y_{40}$ & 37 & $\mathbf{x x}$ \\
\hline & & 1142.3 & $x_{30}$ & 47 & $\mathbf{x}$ \\
\hline & & 1142.3 & $z_{20}$ & 57 & $\mathbf{x}$ \\
\hline & & 1142.6 & $b_{41}$ & 41 & - \\
\hline \multirow{4}{*}{$\mathbf{V}$} & \multirow{4}{*}{1307.6} & 1307.0 & $b_{47}$ & 47 & - \\
\hline & & 1307.3 & $y_{58}$ & 19 & $\mathbf{x x}$ \\
\hline & & 1308.3 & $x_{70}$ & 7 & - \\
\hline & & 1309.7 & $y_{46}$ & 31 & $\mathbf{x}$ \\
\hline \multirow{3}{*}{ VI } & \multirow{3}{*}{1323.7} & 1325.1 & $a_{24}$ & 24 & - \\
\hline & & 1325.2 & $a_{36}$ & 36 & $\mathbf{x}$ \\
\hline & & 1325.3 & $y_{71}$ & 6 & $\mathbf{x}$ \\
\hline \multirow{5}{*}{ VII } & \multirow{5}{*}{1333.1} & 1332.1 & $a_{48}$ & 48 & - \\
\hline & & 1333.1 & $y_{59}$ & 18 & $\mathbf{x x}$ \\
\hline & & 1333.8 & $z_{47}$ & 30 & $\mathbf{x}$ \\
\hline & & 1333.9 & $y_{35}$ & 42 & $\mathbf{x}$ \\
\hline & & 1334.6 & $b_{36}$ & 36 & $\mathbf{x}$ \\
\hline \multirow{3}{*}{ VIII } & \multirow{3}{*}{1356.4} & 1356.1 & $z_{24}$ & 53 & $\mathbf{x}$ \\
\hline & & 1356.9 & $a_{72}$ & 72 & - \\
\hline & & 1357.6 & $a_{37}$ & 37 & $\mathbf{x}$ \\
\hline
\end{tabular}

Table 1: Possible UVPD fragments in a \pm 1.5 Da window. Bold numbers denote Pro residues. Fragments observed previously by UVPD for other charge states are marked with $\mathbf{x}$ when present or $\mathbf{x x}$ when particularly prominent in the reference spectra. 